\title{
EVALUACIÓN, DECOLONIALIDAD Y SABERES OTROS
}

\section{EVALUATION, DECOLONIALITY AND OTHER KNOWLEDGE}

\author{
Julio César Arboleda \\ Dirección Red Iberoamericana de Pedagogía ${ }^{1}$
}

\section{RESUMEN}

El presente número inicia con artículos que conversan en términos de EVALUACIÓN, DECOLONIALIDAD Y SABERES OTROS. LOS primeros 5 permiten advertir abordajes distintos sobre expresiones como saberes otros. Se puede pensar en saberes distintos a los disciplinares, en el caso de la educación precisa abordarse con saberes sobre la realidad socio-cultural, económica-política, eco sistémica-ética/estética (Cardona y otros, 2021); también, saberes nuevos; o saberes invisibilizados, desconocidos o propios de otras culturas, en particular de culturas epistemizadas, colonizadas, violentadas en su saber, en sus conocimientos, cosmovisiones y formas de sentir, vivir, pensar y obrar, de tejer vida comunal. Los siguientes trabajos conectan algunas de estas reflexiones en sus desarrollos sobre temáticas inherentes a Escritura académica, investigación en educación superior, estilos de aprendizaje interculturales, pruebas saber, turismo rural comunitario, y muchos más relacionados con enseñanza y 1 Julio César Arboleda,

Director Red Iberoamericana de Pedagogía,

direccion@redipe.org

https://orcid.org/0000-0002-1572-5384 Profesor investigador de la Universidad de San Buenaventura. Grupos de Investigación Redipe: Epistemología, pedagogía y filosofía. Educación y desarrollo humano, USB. aprendizaje en ciencias naturales, sociales y otras áreas académicas.

\section{PALABRAS CLAVE}

Evaluación, decolonialidad y saberes otros

\section{ABSTRACT}

This issue begins with articles related to subjects such as evaluation, decoloniality and other knowledge. The first 5 articles show us the different approaches for alternative knowledge. People can think of knowledge different from disciplinary knowledge, in the case of education it is necessary to approach it with knowledge about the socio-cultural, economic-political, eco-systemic-ethical/aesthetic reality (Cardona and others, 2021). Besides, it can be new knowledge, or knowledge that is invisible, unknown or typical of other cultures, in particular of epistemized, colonized cultures, violated in their knowledge, worldviews and ways of feeling, living, thinking and acting, of weaving communal life. The following works connect some of these reflections in their developments on topics inherent in academic writing, higher education research, intercultural learning styles, knowledge testing, rural community tourism, and 
many more related to teaching and learning in natural, social and other academic sciences.

\section{KEYWORDS}

evaluation, decoloniality and other knowledge

Los autores coinciden en la necesidad de abogar por el diálogo de saberes y sabidurías pedagógicas, entendidas estas como los conocimientos profundos y amplios que se adquieren $y$ tejen mediante la experiencia educativa, a través de la apertura cognitiva, el encuentro de saberes educativos, posicionamiento político y enunciación del maestro o la maestra; como potenciales que entretejen el encuentro de la otredad, la ruptura de la negación y la voluntad política del quehacer docente (Ahumanda, 2021). Se trata de un diálogo crítico, solidario, ético, comprensivo edificador, diríamos nosotros, abierto a diversas formas de vivencia educativa, que apunte al reconocimiento de otras epistemes, otras formas de saber, de ser, aprender, enseñar, formar, comunicar, de retomar la cultura, de vivir las desigualdades, exclusiones y violencias, la lengua, el territorio y las tecnologías; con sus agenciamientos simbólicos a través de lenguajes múltiples y creativos, ahí el arte, las palabras, las semillas, el agua, la madre tierra, la música y la danza, en los cuales ciertas comunidades sintonizan los lenguajes del movimiento físico, de las fórmulas matemáticas y demás áreas culturales integradas a mundos sociales y culturales específicos. Todos ellos escenarios dialogantes de territorialización y empoderamiento educativo y pedagógico, de memorias y prácticas educativas vivas, alimentadas de sentires comunitarios, que marcan diversas rutas para repensar las asimetrías sociales, económicas, culturales y cognitivas, y también para afrontar los retos contemporáneos. (Ahumada, Unicauca, 2021). Ello aporta para que la educación y también la comunicación interna y externa a esta devenga un saber ser que educa, que transforma al individuo, fortalece y reivindica a las comunidades, identidades y los contextos, sus conocimientos, saberes, cosmovisiones, prácticas, voces, lenguajes y miradas. Sus haceres situados.

Aspectos nutrientes de la educación, tales como la evaluación, precisan de la experiencia y viviencia dialógica, conversacional, subjetiva e intersubjetiva. En virtud a un acercamiento antropológico fenoménico, se asume la evaluación (del aprendizaje) como experiencia humana y antropológica, dinamizada por la subjetividad como territorio existencial del sujeto; como experiencia del conocer, más que de validación del conocimiento como frecuentemente se le asocia a este fenómeno. La experiencia evaluativa es advertida no como un episodio donde se mide y se estratifica a un sujeto, sino que remite a una experiencia de vivencias donde el sujeto encuentre el espacio de potenciarse y se constituya en esa interrelación comunicativa e intersubjetiva con el otro o los otros que coexisten pero que también constituyen y se constituyen en ese mundo de correlaciones de subjetividad e intersubjetividad (Betancourt- Pérez, 2021).

Insiste en que como experiencia humana la evaluación no es un instrumento para medir sino para valorar, para reflexionar, como actitud fenomenológica. Reclama, siguiendo a otros autores, una conciencia participativa en el proceso evaluativo, un sujeto con el potencial de asomarse por encima de las verdades que le sobrevienen, con el desafío de pensar el potencial del presente histórico sobre la base de reflexionar sobre lo vivido, lo habido o lo que no fue y anticipar desde la utopía las profundidades de lo que ha de vivirse o está por venir. Un sujeto capaz de problematizar el sentido de su quehacer, en una actitud que se traduzca en la necesidad de realidad por sobre la organización fija de contenidos. 
En el mismo sentido apuestan por la dignificación de la educación, no sin una perspectiva actuante de comunalidad, que precisa ir más allá de la "modernidad/colonialidad eurocentrada y occidentalizada", óptica vital en virtud de la cual se puede enfrentar creativamente el agregamiento ontológico, epistémico, racial, cultural y político del otro y de lo otro. Una tarea en la que las ciencias de la educación asuman una actitud otra, potencialmente consciente y actuante, si se prefiere decolonizante, que implica un quehacer educativo decolonial, otro, y una hermenéutica para la vida pluriversa, otra. El quehacer decolonial debe expresarse en una actitud de deconstrucción, profilaxis y generación de nuevos conceptos para que las prácticas sociales situadas se carguen de sentido de vida común, interdependiente, evolutiva.

Se requiere, de acuerdo con Ortiz y otros (2021), potenciar la altersofía, un saber otro, no como un nuevo paradigma educativo o modelo pedagógico, sino como una opción, como una forma "otra" de educar, enseñar y evaluar.

Esta rebobinación de la educación y de la pedagogía demanda, lo que en su crítica de resorte intercultural Duque (2021) actualiza una tensión o conflicto epistemológico y ontológico, ontológico-político. El Epistémico se da entre al menos 2 formas de conocimiento: por una parte, la episteme moderna (despótica, eurocéntrica, reduccionista, "superior"), atada a la razón política moderna, y por otra parte las epistemes otras, "inferiorizadas por aquella, en particular las perspectivas decoloniales que reflexionan, reivindican $y$ valoran las cosmovisiones (ninguneadas por el constructo "moderno" como metafísicas, míticas) que resaltan las luchas de las comunidades indígenas, afro y campesinas contra las formas presentes de agregación del otro y lo otro no moderno. Así mismo, el conflicto ontológico, fáctico, entre una ontología moderna, si se quiere transmoderna pero de basamento dualista basada en una política racional impositiva/ positivista/naturalista en materia científica, y una ontología relacional, cosmopolítica, que sustenta a la epistemología de lo ancestral, adversaria al colonialismo de poder, saber y ser, al positivismo, al extractivismo epistémico, espiritual, político y económico; una ontología relacional, ipolítica!, que alcanza a abrazar la dimensión espiritual de la vida, en que se aboga por relaciones más edificantes en un complejo de mundos múltiples y divergentes.

En esa vía va el artículo de Ramírez, A, (2021) en torno a Teorías Decoloniales, en donde llama la atención sobre la pertinencia de acceder a este saber y usarlo en las discusiones actuales y agencias sobre sobre la construcción de currículos y la enseñanza de las áreas culturales desde una mirada emancipadora, y la búsqueda de justicia social en la educación.

Estas perspectivas son relevantes para catalizar y no aplazar el acto ético político de enseñar, aprender y formar educando. Porque, como hemos expresado en varias oportunidades (Arboleda, 2021), una de las tareas de la educación es generar escenarios para despertar en los educandos consciencia reflexiva, crítica, generativa y edificante sobre sí mismos, su relación con el mundo y la vida pluriversa. Veamos una síntesis de cada artículo.

\section{LA SUBJETIVIDAD Y LA INTERSUBJETIVI-}

DAD: DOS VECTORES EN LA EVALUACIÓN COMO FENÓMENO ANTROPOLÓGICO. Jorge H. Betancourt Cadavid, Johann Enrique Perez. Universidad de San Buenaventura. Artículo de investigación. Asume la subjetividad e intersubjetividad como nociones fenomenológicas al interior de la evaluación en la educación, para interpretar la vida humana inherente a ella. Lo hace desde estos dos vectores, fundamentando una búsqueda de elementos que desde la antropología fenomenológica consolidan dentro del campo de la pedagogía una reflexión filosófica en torno a la 
constitución del sujeto y los otros de su entorno en la evaluación, describiendo la correlación entre las operaciones o efectuaciones constituyentes del mundo y lo efectuado en estas; se trata de la alusión a un Yo que cobra su sentido de las efectuaciones de la conciencia, pero que no es un Yo solo en el mundo, como una mónada incomunicable, sino en presencia e interacción con otro u otros con los que comparte un mundo, como condición de posibilidad de que un Ego pueda interactuar con el Otro para concretar su humanidad. Esta búsqueda se describe en torno a cuatro apartados que constituyen el cuerpo del articulo y cada uno argumenta la premisa de que la evaluación es una experiencia humana, por ende, un fenómeno antropológico que supera la reiterativa y afincada descripción de la evaluación como dispositivo.

\section{LOS MODELOS DIDÁCTICOS EN LA FORMACIÓN DE DOCENTES UNIVERSITARIOS, LA EMERGENCIA DE $Y$ AMBIENTAL) EN PANDEMIA DE LA} COVID-19. Claudia María Cardona- Edwin Rivas Trujillo - María Ramírez Sánchez, Universidad Distrital Francisco José de Caldas. Artículo de investigación. Presenta los saberes-otros que emergen como importantes en Pandemia de la Covid-19 y que hacen parte de la formación del Docente Universitario. El inicio es el modelo pedagógico/didáctico de (Shulman, 1987 -PCK), y la fusión de muchos que mezclan de manera independiente esos saberes-otros, como el de (Mishra \& Koehler, 2006 -TPACK y el de Zhou, 2015- EPACK). A manera de escenario apuesta para la educación del siglo XXI situada en un horizonte de nuevas realidades globales, nos ubica ante retos inéditos, por ejemplo, 'el tecnológico' y 'lo ambiental', esos llamados los saberes-otros, que, desde la mirada de una inclusión de una educación superior, compromete a un cambio de paradigma que favorece la interdisciplinariedad y el aprendizaje investido.

DECOLONIZAR LAS CIENCIAS DE LA EDUCACIÓN: PEDAGOGÍA, ALTERSOFÍA Y HACER EDUCATIVO DECOLONIAL. Alexander Ortiz Ocaña, Ana Maloof Díaz y Habib Mejía Porto, Universidad del MagdalenaUPC. Artículo de reflexión generativa. Pone de presente la urgencia de configurar nuevas formas de enseñar sustentadas en el saber del Otro, considerado inferior, y no sólo en la pedagogía moderna/colonial, considerada superior. Sugerimos decolonizar las ciencias de la educación mediante la Altersofía y el Hacer Decolonial. Asume la Altersofía como una forma "otra" de educar, para el desprendimiento de la pedagogía moderna/colonial. Analiza para qué una didáctica "otra". Caracteriza el hacer educativo decolonial como proceso formativo decolonizante, para desobedecer a la didáctica USA-eurocéntrica, mediante acciones y huellas de aprendizaje: contemplar comunal, conversar alterativo y reflexionar configurativo, que caracterizan la vocación educativa decolonial.

\section{APUESTAS DESDE LA EDUCACIÓN POPULAR. Saberes y Sabidurías Pedagógicas: Encuentro de la palabra y} las experiencias educativas. Magda Alicia Ahumada, Universidad del Cauca. Artículo de reflexión generativa en torno a las experiencias de la Educación Popular en el Cauca- Colombia, desde el diálogo de saberes y sabidurías pedagógicas, como escenarios transformadores de la educación local. Vivencias educativas locales que abre las puertas al repensar de lo solidario, los ejercicios colaborativos, la dignificación de la vida, y los innumerables retos de lo tecnológico y la virtualidad, en tiempos de pandemia.

THE DECOLONIAL OPTION: IMPLICATIONS FOR THE CURRICULUM OF FOREIGN LANGUAGE TEACHER EDUCATION PROGRAMS. Alexánder Ramírez Espinosa, Universidad del Valle, Cali - Colombia. Artículo de reflexión generativa. Se deriva de las 
discusiones y sesiones argumentativas que se dieron en el marco de un seminario doctoral sobre Teorías Decoloniales. Propone un doble objetivo: por un lado, presentar sus reflexiones en torno a la literatura revisada y discutida a lo largo del seminario, y por otro, arrojar luz sobre cómo dicha literatura puede tener un impacto positivo en los currículos de programas de formación de dicentes en lengua extranjera. Los argumentos que el autor presenta aquí son producto de las discusiones con colegas y docentes; en ese sentido, las ideas aquí presentadas están abiertas al escrutinio y al diálogo por parte de otros académicos. Se espera que este artículo contribuya a las discusiones actuales sobre la construcción de currículos y la enseñanza de lenguas extranjeras desde una mirada emancipadora, y la búsqueda de justicia social en la educación.

\section{ESCRITURA ACADÉMICA DE ESTUDIANTES INDÍGENAS EN LA UNIVERSIDAD. Margareth}

manera adecuada las convenciones del discurso académico. Entre los aspectos que deben priorizarse para alcanzar la literacidad académica están la ampliación del léxico en español y del vocabulario técnico, la identificación y uso de recursos semánticos y discursivos para cohesionar y dar coherencia a las ideas del texto, la construcción de textos con la superestructura requerida y la incorporación de la polifonía con diferentes propósitos y con las convenciones establecidas para ello.

INVESTIGACIÓNENEDUCACIÓN SUPERIOR: FACTOR CLAVE EN LA CONSTRUCCIÓN DEL CONOCIMIENTO.. Wlamyr Palacios AlvaradoByron Medina Delgado- Jorge Gómez Rojas, Ufps- Universidad del Magdalena. Resalta la investigación en la educación superior como factor clave en la construcción y generación de conocimiento. Concluye que las instituciones de educación superior están obligadas a desarrollar planes, programas y estructuras curriculares centradas en la investigación, con el propósito de que estudiantes desarrollen competencias científicas para aprender a aprender, construir conocimiento y resolver los problemas del entorno socio-económico.

\section{ONTOLOGICAL BASE MODELS MACHINE-} TO-MACHINE M2M APPLIED TO THE INTERNET OF THINGS IOT. Mauricio Orlando Bermúdez Amaya- Salcedo Parra, Octavio José- Rodríguez Miranda, Juan Pablo. UFJDC- UNIVERSIDAD NACIONAL DE COLOMBIA. Artículo de investigación. Propone una evaluación sobre los diferentes modelos ontológicos que consideran el M2M y el loT en simultáneo, reconociendo la capacidad sintáctica y semántica de la interoperabilidad de dichos dispositivos, a partir del estudio de los esquemas básicos en mención, e identificando sus propiedades más destacadas según la métrica Calidad de Servicio QoS, obteniendo la ontología oneM2M como la más adecuada. 
EVALUATION OF THE USE OF THE NATIONAL ACADEMIC NETWORK OF ADVANCED TECHNOLOGY (RENATA) IN COLOMBIA. Perdomo Duarte, Julian Camilo- Salcedo Parra, Octavio José - Sánchez Céspedes, Juan Manuel, Universidad Nacional De Colombia- Udfjc. Artículo de investigación. La importancia académica y científica del artículo radica en discutir el problema de que las redes NRAN no se aprovechen como herramientas científicas que pueden tener un efecto dominó al impulsar el uso de dichas herramientas en diferentes centros de educación e investigación del más alto nivel. Se concluye que el modelo de conectividad en las redes asociadas, es decir, las redes universitarias de todas las ciudades importantes dentro de RENATA, no aprovechan de manera efectiva las ventajas de la capacidad de ancho de banda, probada con 0.039 Gbps.

\section{ESTILOS DE APRENDIZAJE INTERCULTU-}

RALES (SENSIBILIDAD INTERCULTURAL)

$21 . \quad$ Y MODALIDADES EN LA CARTA ARGUMENTATIVA EN ESTUDIANTES BILINGÜES DE LICENCIATURA EN LENGUAS MODERNAS DE UNA UNIVERSIDAD COLOMBIANA Y UNA FRANCESA. Neira Loaiza Villalba, PhD., Universidad del QuindíoClaudia Elizabeth Ortiz Ruiz, Mg., Universidad del Valle. Artículo de investigación. Desde las teorías del bilingüismo escolar y las ciencias del lenguaje, este estudio de caso cualitativo describe cómo los estilos de aprendizaje, ligados a la sensibilidad intercultural, se reflejan en la competencia argumentativa escrita bilingüe en términos de las modalidades discursivas en una carta argumentativa en L1 y L2 en estudiantes bilingües de licenciatura en lenguas modernas (francés y español LE) de dos universidades, una colombiana y una francesa. En general, los resultados muestran un perfil de estilo de aprendizaje en donde hay una presencia significativa del estilo reflexivo y del estilo verbal y un equilibrio entre lo sensorial y lo intuitivo, y entre lo secuencial y lo global. En cuanto a la sensibilidad intercultural, se evidencia una actitud favorable y neutra alta que facilita la interacción intercultural. A partir del análisis de retórica contrastiva intra e interindividual, se encuentra que las modalidades sobresalientes son la epistémica, la alética y la deóntica que validan las representaciones desde lo objetivo, lo subjetivo y/o lo institucional.

\section{ENSEÑANZA - APRENDIZAJE DEL INGLÉS} BAJO LAS POLÍTICAS DEL MINISTERIO DE EDUCACIÓN EN COLOMBIA: VOCES DESDE EL AULA DE CLASE. Margarita M. López Pinzón- Odilia Ramírez ContrerasMauricio Arango Vélez, Universidad de Caldas, Manizales, Colombia. Artículo de investigación. Describe las percepciones de profesores, estudiantes y directivos sobre la enseñanza y aprendizaje del inglés en colegios públicos bajo los lineamientos del Ministerio de Educación, específicamente el Currículo Sugerido (CSI) y los Derechos Básicos de Aprendizaje (DBA). Los participantes reportaron percepciones positivas acerca de la implementación de estas políticas en lo relacionado con el uso apropiado de los enfoques metodológicos, los materiales y recursos, los contenidos temáticos y las estrategias de evaluación en sus aulas de clase; sin embargo, también sostuvieron que se requiere darle continuidad a los programas de bilingüismo para alcanzar los objetivos de nación en esta asignatura y recibir apoyo permanente de las autoridades locales y nacionales tanto en inversión como en acompañamiento pedagógico pues estas políticas se consideran una forma de brindar a los estudiantes la oportunidad mejorar los niveles de proficiencia en inglés en el nivel de educación básica secundaria y media.

FORTALECIMIENTO DEL PENSAMIENTO VARIACIONAL A TRAVÉS DE LA GAMIFICACIÓN Y LA TEORÍA DE LA OBJETIVACIÓN. Ingrid Milena Parra Arenales, Wilson Andrés Martínez-Delgado, Élgar Gualdrón-Pinto, Universidad Autónoma 
de Bucaramanga. Universidad De Pamplona. Artículo de investigación. Orientada a fortalecer el pensamiento variacional de los estudiantes a través de una estrategia didáctica basada en la gamificación como elemento motivacional y adaptando las actividades a desarrollar según lo planteado en la teoría de la objetivación propuesta por Luis Radford. El análisis de los resultados a través de las categorías de análisis refleja que tanto el factor motivacional como el desarrollo del pensamiento variacional fueron afectados favorablemente tras la implementación de la estrategia didáctica.

\section{REFLEXIONES ACERCA DE UN PROGRAMA} DE ELE: DESDE LA PERSPECTIVA DE LOS ESTUDIANTES. Numas Armando Gil OliveraDayson David Ahumada Ebratt, Universidad Del Atlántico- Universidad Del Norte. Artículo de investigación. Describie las perspectivas de los estudiantes de un programa de ELE llamado Didáctica de la enseñanza del español como lengua extranjera perteneciente a la carrera de Licenciatura en Idiomas Extranjeros de la Universidad del Atlántico, Barranquilla, Colombia. Haciendo especial énfasis, en el alcance de los objetivos de aprendizaje que se plantea tal asignatura. Entre sus resultados reportó que la gran mayoría de los estudiantes si lograba alcanzar los objetivos de aprendizaje porque mostraban un amplio manejo de aspectos como los métodos y enfoques que se promovían en la asignatura. Este tipo de resultados, si bien no son absolutamente generalizables, si son un excelente punto de inicio para aquellos docentes de ELE que quieran incursionar y profundizar en estás temáticas.

CREACIÓN DE UN HÉROE EN INGLÉS PARA EDUCANDO HOSPITALARIO: UN ESTUDIO DE CASO. Gersson Joaquín Buitrago Garcia - Juan Camilo Villalobos Quintero

- Astrid Ramirez Valencia, Universidad Distrital Francisco José de Caldas. Artículo de investigación. Estudio de caso sobre el análisis del proceso de arteterapia, estrategia implementada en un educando hospitalario, quien por medio de la creación del héroe en inglés y su experiencia hace un manejo del duelo. Para el grupo de sesiones se consideran diferentes teorías, Kolb (2015), Romero (2014), Klein (2006), entre otros, donde es el enfoque del aprendizaje experiencial el protagonista de las sesiones, facilitando el proceso de creación por medio del inglés, además del manejo del duelo. La interpretación da cuenta de resultados donde los factores socio-académicos y situaciones fluctuantes son determinantes a lo largo de la investigación.

\section{REVISIÓN DE PARES EN UN CURSO DE ESCRITURA CREATIVA EN INGLÉS: FOCO Y POSICIONAMIENTO DE LOS REVISORES.}

Sol Colmenares Rodríguez, Alexánder Ramírez Espinosa, Universidad del Valle, CaliColombia. Artículo de investigación. Describ las revisiones hechas por estudiantes y establece, por un lado, en qué aspectos del texto se enfocan, y por otro cómo se posicionan en su rol de revisores. Los resultados sugieren que, en este caso, las revisiones trascendieron el foco tradicional de la corrección lingüística y se centraron en aspectos globales y estilísticos del texto tales como la construcción de personajes y la construcción de audiencia.

\section{APRECIACIÓN EN LOS RESULTADOS DE LAS PRUEBAS SABER PRO. Derly Francedy} Poveda Pineda- José Eriberto Cifuentes Medina- José Antonio Chacón Benavides, Universidad Pedagógica y Tecnológica de Colombia. Artículo de investigación. Analiza los resultados de las pruebas Saber Pro, para el año 2020, de los estudiantes pertenecientes al programa de Licenciatura en Educación Básica con énfasis en Matemáticas, Humanidades y Lengua Castellana, de la Universidad Pedagógica y Tecnológica de Colombia - UPTC. El estudio está correlacionado a las competencias específicas de la formación de licenciados; 
competencias que se enmarcan en: Enseñar, Evaluar y Formar; componentes propios de un profesional en educación y con especificidad en educación básica. Realiza la comparación entre el programa, la institución, la sede y el grupo de referencia, que permitió consolidar un estudio descriptivo y una aproximación a los resultados obtenidos por los estudiantes del programa.

\section{PERFIL AFECTIVO-EMOCIONAL EN PRO-}

\section{FESORES DE CIENCIAS ADMINISTRATIVAS} HACIA CIENCIAS BÁSICAS . Henry de Jesús Gallardo-Pérez, Mawency VergelOrtega, Carlos Sebastián Gómez Vergel. Artículo de investigación. Dirigido a identificar las emociones en los profesores de ciencias empresariales y sus factores determinantes. Los profesores de ciencias empresariales del mismo tipo de institución tienen competencias y manejo de emociones similares en comparación con los de otra institución educativa; se incorporaron variables predictoras de los niveles: institución educativa y factores académicos del profesor de ciencias empresariales y su interrelación. La investigación concluye que existen tres perfiles de profesores que orientan en programas de ciencias empresariales: perfil asociado a la formación profesional y a la percepción hacia las ciencias básicas, así mismo, el modelo multinivel de emociones es adecuado para describir la relación entre las variables predictoras, las actividades orientadas a la enseñanza y el fomento de ambientes de aprendizaje que desarrollen competencias en emprendimiento y gerencia de empresa en los estudiantes de educación superior para promover el aprendizaje significativo y fortalecer la estrategia institucional basada en el manejo de las emociones de los profesores de ciencias empresariales.

ENSEÑANZA VIRTUAL DE LAS CIENCIAS EMPRESARIALES: RETOS Y DESAFÍOS. Henry Orlando Luna Pereira, Gerson Rueda Vera, William Rodrigo Avendaño Castro, UFPS. Artículo de investigación. Determina los retos y desafíos de las recientes iniciativas de enseñanza virtual como resultado de la pandemia COVID-19 desde la perspectiva de los profesores universitarios de ciencias empresariales de una universidad pública de la ciudad de Cúcuta (Colombia). Se concluye que el reto es usar herramientas tecnológicas y al mismo tiempo hacerlo en la gestión y organización de la enseñanza, construcción de comunidades de aprendizaje, comunicación y estrategias de enseñanza.

PERSPECTIVAS DEL TURISMO RURAL COMUNITARIO EN ÁREAS PROTEGIDAS; UNA REVISIÓN BIBLIOGRÁFICA 2010-2020. Castro-Garzón, Hernando, López Toledo, Luz Sofia, Universidad de los Llanos- Rodríguez Miranda, Juan Pablo, Universidad Distrital Francisco José de Caldas. Bogotá, Colombia. Artículo de investigación. Establece un resumen de la relación del turismo rural comunitario en las áreas protegidas y la sinergia con los ecosistemas. Se realizó una revisión de la literatura especializada en diferentes bases de datos y se encontraron resultados de orden mundial, regionales y locales. Se analizó la conceptualización y el abordaje, en las áreas protegidas.

\section{ANÁLISIS DE LOS ESTABLECIMIENTOS} COMERCIALES EN LA CIUDAD DE VILLAVICENCIO FRENTE A LOS IMPACTOS NEGATIVOS DE TIPO SOCIOECONÓMICO. Castro-Garzón, Hernando, Hernández Criado, Juan Carlos, Sánchez Céspedes, Juan Manuel, Unillanos, UFPS- UDFJC. Artículo de investigación. Realiza una caracterización de los establecimientos para medir el impacto de los factores socioeconómicos que los afectan para que así se puedan formular estrategias para mitigar los efectos que estos producen. En las conclusiones se resaltó que los comerciantes percibían el factor económico como el de mayor impacto negativo a causa de la crisis por la pandemia; entre los conceptos sociales, se 
determinó que la inseguridad a pesar de ser un factor de vieja data los sigue afectando, también propusieron una mayor participación por parte del gobierno seccional, estrategias publicitarias para el centro, programas de rehabilitación para los habitantes de calle y reubicación de los vendedores ambulantes.

\section{TIPOLOGÍA Y VECINDADES EN HITOS} SAGRADOS DE LA CIUDAD DE SAN JOSÉ DE CÚCUTA-COLOMBIA Yannette Díaz Umaña, Mawency Vergel Ortega Julio Alfredo Delgado Rojas. Artículo de investigación en torno al concepto topológico de vecindad, donde una superficie es vecina de otra con conexiones o conectividades unidos por signos y símbolos, permiten dar continuidad a interpretaciones tipológicas, relacionadas por su devenir histórico, representación estilística y variables afín a la altura de las edificaciones. Por consiguiente, se consolida la noción de lugar y el concepto de vecindad. Los datos obtenidos sin considerar las magnitudes, han permitido hacer un análisis transversal de estas torres con el fin de analizar su topología, sus relaciones y asonancias de manera que se identificó ciertas cualidades arquitectónicas y urbanas.

IDENTIDAD PROFESIONAL DOCENTE EN TIEMPOS DE CRISIS: RELEVANCIA DE LA EMPATÍA EN LA PRÁCTICA PEDAGÓGICA, Ibeth Morales-Escobar- Marisol Correa Londoño- Arnaldo Peinado Méndez, Universidad de Córdoba, Facultad de Educación y Ciencias Humanas, Montería, Colombia. Artículo de investigación. Analiza, desde las narrativas de profesores y estudiantes, las transformaciones que se han dado en la identidad docente en el contexto de la pandemia y cómo estas se constituyen en fundamento para la agenda de formación de maestros en la década que vivimos. Se realiza desde una perspectiva cualitativa con método de documentación narrativa, para conocer las vivencias de los docentes y estudiantes desde su propia voz y reconstruir los saberes, actitudes, en definitiva, la experiencia que tienen los docentes de su propio trabajo en el marco de esta crisis sanitaria mundial. El análisis permite inferir que la empatía se reconoce como una actitud profesional que ha tenido poder transformador en los sentimientos y acciones de los estudiantes, y que permitió acompañar y continuar con los procesos de enseñanza y de aprendizaje.

"PARCEROS": UN PROGRAMA PARA LA PREVENCIÓN E INTERVENCIÓN DEL ACOSO ESCOLAR (BULLYING) EN COLOMBIA. Herrera-López, Mauricio; Lagos, Alejandra; Villota, Yaqueline; Pantoja, Diana, Figueroa, María Fernanda, Universidad de Nariño. Artículo de investigación. Sobre la implementación de un programa de intervención para prevenir y mitigar el bullying, a partir del fortalecimiento de habilidades socioemocionales, desarrollado en una institución educativa oficial del municipio de San Juan de Pasto, sur de Colombia. Los resultados cuantitativos sugieren una mayor disminución en la prevalencia del rol de agresor y agresor-victimizado, en comparación a un menor cambio en el rol de victimización, a su vez los resultados cualitativos evidencian un incremento en el reconocimiento de la dinámica del acoso escolar y un mayor uso de las habilidades socioemocionales por parte de los participantes en el contexto educativo, lo cual da cuenta del mejoramiento de las relaciones interpersonales entre los estudiantes y de la promoción de una sana convivencia.

INTERPRETAR LA OBRA DE EDUARDO RAMÍREZ VILLAMIZAR: CONFIGURACIONES GEOMÉTRICAS BI Y TRIDIMENSIONALES. Fabián Alfredo Mena Uscategui - Javier Alberto Mariño Díaz - Mawency Vergel Ortega Artículo de investigación. Dirigidao a generar un proceso de enseñanza - aprendizaje a partir de la interacción grupal entre docentes y estudiantes de arquitectura de la Universidad 
de Pamplona para el estudio de los atributos conceptuales, geométricos y tecnológicos de la obra del maestro Eduardo Ramírez Villamizar hacia la realización de reinterpretaciones creativas a través de perspectivas sensibles de autores teóricos contemporáneos. Para ello se realiza un análisis de los atributos sensibles, geométricos y tecnológicos de la obra del maestro Eduardo Ramírez Villamizar en jornadas de socialización de trabajo, dialogo y autocritica. Resultado muestra que las experimentaciones, permiten potenciar la creatividad e innovación en los jóvenes.

TRANSICIÓN DE LA EDUCACIÓN PRIMARIA A LA SECUNDARIA. UNA MIRADA DESDE EL DOMINIO AFECTIVO HACIA LAS MATEMÁtICAS. Raúl Prada Nuñez, Luis Fernando Mariño, Cesar Augusto Hernández Suarez. Artículo de investigación. Sobre la influencia del componente afectivo como un factor determinante en el éxito o fracaso de las matemáticas, con la consideración de si este rechazo se debe a la labor del docente o a los contenidos de la asignatura. Los resultados permiten identificar la influencia que tienen las creencias, las actitudes y las emociones hacia las matemáticas sobre el desempeño académico de los estudiantes en la disciplina. Al tiempo que se reconoce el rol del docente como un elemento crucial en la construcción de actitudes positivas respecto a la asignatura.

\section{PERCEPCIONES SOBRE EL DESARROLLO} DE COMPETENCIAS INVESTIGATIVAS EN ESTUDIANTES DE SEMILLEROS DE INVESTIGACIÓN. Cesar Augusto Hernández Suárez, Audin Aloiso Gamboa Suárez, Raúl Prada Núñez. Artículo de investigación. Muestra los resultados de un estudio que buscó identificar las percepciones que tienen estudiantes de cinco semilleros de investigación sobre el desarrollo de competencias investigativas desde su experiencia en la universidad. Logra revelar que gran parte de los estudiantes utilizan con mayor frecuencia las tecnologías digitales para la búsqueda de información relevante para sus trabajos de investigación y muestran un manejo de las bases de datos especializadas para esta búsqueda.

\section{GESTIÓN DE RESIDUOS LIQUIDOS:} análisis de la generación de AVUS, en restaurantes y cafeterías de la comuna cuatro de Villavicencio (Meta, Colombia). Castro-Garzón, Hernando, Ávila Sierra Héctor Manuel, Salcedo Parra, Octavio José, Unillanos. Santo Tomás y UDFJC. Artículo de investigación. Analiza la generación de aceite vegetal usado (AVU) en restaurantes y cafeterías de 30 barrios de la comuna 4 de Villavicencio Meta, y se plantean alternativas para el manejo adecuado y disposición final del residuo, mediante una investigación de tipo exploratoria-descriptiva, que permite identificar zonas de mayor generación del residuo, formas de conducta frente a la problemática estudiada, cantidad y frecuencia de generación y los hábitos de gestión del residuo, información que fue recolectada con la aplicación de una encuesta semiestructurada compuesta por 18 preguntas. Los resultados indicaron que existe gran desconocimiento de las alternativas de gestión del residuo, adicional a esto, más del $40 \%$ de las unidades encuestadas son conscientes de los impactos ambientales causados al verter aceites a las fuentes hídricas o los daños a la salud que genera reutilizar esta sustancia, por otro lado teniendo en cuenta la visita a la corporación autónoma regional del Meta, y la recolección de información de fuentes secundarias y primarias, se pudo afirmar que existe un conjunto de regulaciones a nivel nacional e internacional que hablan de la importancia del desarrollo sostenible de manera imprecisa o vaga frente a lo que el aceite usado de cocina respecta, pero hace falta en el ámbito local la aplicación del principio para el adecuado manejo del aceite vegetal usado en Colombia, no hay normativa que busque el adecuado manejo del aceite vegetal usado 
y su potencial concreto como materia prima de otros productos, las alternativas actuales están basadas en las practicas informales de recolectores, no existe una estrategia municipal organizada que este enfocada en la gestión de este residuo peligroso.

EFECTOS AMBIENTALES: análisis de los impactos presentes en un cuerpo de agua urbano en la ciudad de Villavicencio (Meta, Colombia), Castro-Garzón, Hernando; Sánchez Céspedes, Juan Manuel; Rivas Trujillo, Edwin. Artículo de investigación. Dirigido a establecer los impactos generados por quienes residen en la margen del cuerpo de agua denominado caño la Cuerera, en el trayecto correspondiente al barrio Macunaima, en la ciudad de Villavicencio Meta, Colombia. Dando como resultado que la principal fuente de impacto negativo del afluente son los vertimientos domésticos, dado que son los de mayor recurrencia a lo largo del cauce.

PROTOTIPO ANATÓMICO PARA EL APRENDIZAJE DEL VII PAR CRANEAL QUE SIMULA UN IMPULSO NERVIOSO EN RELACIÓN CON LA BASE DEL CRÁNEO. Luis Felipe Muñoz, Janneth Zúñiga, Sonia Osorio, Universidad del Valle. Artículo de investigación. Sobre el diseño de una herramienta pedagógica que permita observar la ubicación y recorrido del VII par craneal junto con sus impulsos nerviosos. A partir de imágenes en diferentes planos anatómicos del cráneo humano se diseñó un armazón con la estructura característica del mismo. Para dar volumen y forma a cada estructura ósea se utilizó porcelanicrón, y los accidentes óseos de los huesos de la base del cráneo y forámenes fueron moldeados a mano. Finalmente, se construyó el VII par craneal, representando los impulsos nerviosos por medio de pulsos eléctricos con una secuencia led. Se logró realizar una modelo 4D del cráneo que incluye el recorrido nervioso derecho del VII par craneal desde su origen hasta el lugar de inervación. Así mismo, se observa la simulación del estímulo nervioso de las diferentes fibras nerviosas. El aprendizaje de los pares craneales desde la creación de un modelo anatómico proporciona conocimiento anatómico integrado, ya que facilita la ubicación y dimensión de estructuras difícilmente apreciables en piezas reales. Esta estrategia también brinda al estudiante una representación mental de las vías nerviosas y cómo estas se pueden ver afectadas en ciertas condiciones patológicas o traumáticas, lo que contribuirá de forma significativa a la futura práctica clínica.

\section{METODOLOGÍA PARA LA IDENTIFICACIÓN DE BARRERAS EN LOS PROCESOS DE CERTIFICACIÓN EN INOCUIDAD ALIMENTARIA: ESTUDIO DE CASO DE LA INDUSTRIA CÁRNICA DE} BOVINO DE LA CIUDAD DE CÚCUTA. Naydu Judithjácome Castilla Eduardo Luis Jacome Castilla Y Erwin Hernando Jacome Castilla. Artículo de investigación. Muestra los resultados de un proceso de investigación orientado a analizar las barreras en la implementación de normas internacionales en la fabricación de productos cárnicos de la ciudad de Cúcuta. Desde los preceptos establecidos por la International Organización for Standadization, con la norma "Garantía de la seguridad alimentaria", se estudiaron los principales impedimentos que presentan las empresas de Cúcuta en el cumplimiento de las condiciones para obtener la certificación internacional.

TRAZABILIDAD DE LA DEMANDA DEL PROGRAMA DE CONTADURÍA PÚBLICA. Yair Rolando Casadiego Duque, Carlos Fabian Rico Rojas, Raúl Prada Núñez. Artículo de investigación. Se orienta a proveer información para el cumplimiento de las condiciones de calidad exigidas por el Mineducación, en relación a los análisis por periodos académicos de los siguientes indicadores de los programas 
similares de referencia: Personas inscritas, admitidas y matriculadas en primer curso; total de matriculados y graduados; tasas de deserción por cohorte y por periodo académico. Los resultados permiten concluir el estado de los programas académicos de Contaduría Pública desde una visión nacional, para luego aterrizarla a lo regional y finalizar con un análisis institucional del programa.

SATISFACCIÓN ESTUDIANTIL CON EL USO DE OBJETOS DE APRENDIZAJE APP EN BIOQUÍMICA EN LA CARRERA DE MEDICINA DE LA UNIVERSIDAD NACIONAL DE CHIMBORAZO (UNACH). María Angélica Barba Maggi Lucila Jazmín De la Calle Andrade-Yisela Carolina Ramos Campi-Erika Valeria Calderón Barba- Gustavo Eduardo Fernández Villacrés- Diana Cristina Aguilar Marcillo Ecuador. Artículo de investigación. Analiza el nivel de satisfacción con el uso de los Objetos de aprendizaje - app con tecnología Bioquímica, en la temática de Hemoglobina y Mioglobina, en los estudiantes de los primeros semestres de la carrera de Medicina de la UNACH. Los resultados fueron analizados con la herramienta estadística Excel, evidenciándose que la satisfacción estudiantil después del uso de los Objetos de aprendizaje - app con tecnología Android supera el $90 \%$ de acuerdo y muy de acuerdo, en la escala valorativa más alta.

\section{VALUE-ADDED MODEL OF BASIC MATHEMATICS-PHYSICS TRAINING IN ENGINEERING STUDENTS.}

Henry de Jesús Gallardo Pérez ${ }^{1}$ Mawency Vergel Ortega ${ }^{2}$ Marling Carolina Cordero Díaz Universidad Francisco de Paula Santander, San José de Cúcuta, Colombia. Artículo de investigación, dirigido a determinar el valor agregado del trabajo académico de la Universidad Francisco de Paula Santander en el desarrollo del pensamiento físico-matemático en los estudiantes de ingeniería y la estimación

de un modelo matemático que permita su valoración. Se obtuvieron datos primarios de una prueba aplicada en 2018 y otra similar aplicada en 2019, calificada mediante la teoría de respuesta al ítem. Se compararon los resultados y se evaluaron las diferencias para estimar la contribución efectivamente realizada por la universidad.

\section{ESTABLECIMIENTO DE LOS FACTORES} DE GESTIÓN HUMANA APLICADOS EN UN GRUPO DE ESTABLECIMIENTOS DE REFACCIÓN DE AUTOMOTORES EN VILLAVICENCIO-META. Castro-Garzón, Hernando, Hernández Criado, Juan Carlos, Rivas Trujillo, Edwin. Unillanos, UFPSUDFJC . Artículo de investigación. Analiza el comportamiento que tienen los establecimientos dedicados al comercio y reparación automotriz o de autopartes para identificar las fortalezas como las debilidades presentes, así mismo las oportunidades junto con las amenazas, por otro lado, se caracterizaron los puntos de trabajo, se describieron los procesos salariales, de prestaciones sociales, también se realizó un análisis de los resultados obtenidos. La intervención permitió detallar, además de actualizar, como se encuentra el sector automotriz en tanto a temas salariales como de prestaciones sociales; adicionalmente ayudó a los empresarios y comerciantes a reflexionar o informarse sobre las cuestiones laborales legales en el país; por otra parte, recopilar información tendiente a medir el estado, así como el comportamiento del sector en general. Dentro de las conclusiones más relevantes se encontró que casi la mitad de establecimientos no afilian ni pagan prestaciones sociales legales.

PROPUESTA DE MODELO CONCEPTUAL PARA MIPYMES $Y$ EMPRENDEDORES COMO HERRAMIENTA DE APOYO PARA CONTRARRESTAR OBSTÁCULOS DEL MERCADO. Castro-Garzón, Hernando= Hernández Criado, Juan Carlos- 
Rivas Trujillo, Edwin. Artículo de investigación. En torno a una herramienta guía para los futuros emprendedores, aunque también puede ser usada por empresarios, dado que llevará a que considerar poner en práctica referentes tendientes a la supervivencia organizacional. Se obtuvo como resultado la elaboración de un modelo conceptual que permite al usuario obtener una visión de los principales obstáculos del mercado, con la manera efectiva en que puede contrarrestarlos, como también los factores de éxito y sus claves para lograrlo. El modelo representa los factores que tienen un impacto, que puede ser positivo o negativo, según sea el manejo de la empresa, además, contiene los parámetros que podrá seguir cada organización para fortalecer o implementar dentro de sus procesos.

CONVERGENCIA DE SABERES EN LA FORMACIÓN DEL TRABAJO SOCIAL CONTEMPORÁNEO. Milton Alier Montero-
Ferreira, Laura Nataly Galvis Velandia, Maritza Carolina Jaimes Márquez, Facultad de Educación, Artes y Humanidades. Artículo de investigación. Hace parte de los resultados de la investigación "Aproximación teóricometodológica al modelo de convergencia en Trabajo Social, aportes y desafíos al desarrollo de la profesión en el siglo XXI"; se desarrolló desde el pensamiento complejo; En los resultados que se presentan, se destaca la categoría de renovación permanente en la formación disciplinar, desde donde se priorizaron las siguientes subcategorías: formar en la relación global y local, la importancia de articular saberes, el desafío de la investigación formativa y la necesidad del pensamiento crítico.

ESCENARIOS URBANOS EN LA OCUPACIÓN COMERCIAL DE LOS ALREDEDORES DEL CENTRO COMERCIAL LAS MERCEDES EN LA ZONA CÉNTRICA DE CÚCUTA. Yannette Díaz Umaña, Julio Alfredo Delgado Rojas, Mawency Vergel Ortega. Artículo de I investigación sobre la apropiación del espacio público de la zona céntrica de la ciudad de San José de Cúcuta. Se sustentó en un estudio etnográfico que permitió la indagación mediante la entrevista de profundidad y la observación, donde se alcanzó el acercamiento a las historias de vida de las personas dedicadas a la actividad informal en el espacio público de los alrededores del centro comercial las Mercedes. Así mismo se examinó el concepto de ocupación comercial y de apropiación del espacio público, para precisar las formas en la que estas personas generan los vínculos con el espacio público, lugar que han escogido para realizar su trabajo informal.

\section{CARACTERIZANDO LA RESOLUCIÓN} DE PROBLEMAS DE FÍSICA DESDE EL ALGEBRA LINEAL $Y$ ESTUDIANTES DE INGENIERÍA. Luis Fernando Mariño, Rosa Virginia Hernández, Víctor Julio Useche Arciniegas, UFPS. Artículo de investigación. Se centra en dar respuesta a la pregunta, ¿cómo son las estrategias para resolver problemas de física que involucran sistemas de ecuaciones lineales, manifestadas por un grupo de estudiantes de ingeniería de sistemas? Los resultados, permitieron elaborar una caracterización de las estrategias para resolver problemas de física, como un flujo de acciones que van desde interpretar, conjeturar, formalizar, resolver, verificar y reflexionar.

\section{DESARROLLO DE UNA APLICACIÓN INFORMATICA COMO AYUDA EN LA DOCENCIA DE LA ASIGNATURA PROCESOS INDUSTRIALES. Milton Fabián Coba} Salcedo' Félix Joaquín Lozano-Cárdenas, Erika Alejandra Maldonado Estévez. Universidad del Atlántico, Ufps. Artículo de investigación. Expone una aplicación informática para la automatización del modelado grafico como ayuda en la docencia de la asignatura Diseño Mecánico en la carrera de Ingeniería Mecánica. Se ha integrado mediante la interfaz de programación de aplicaciones (API) del 
software de modelado grafico Solidworks, una herramienta que permite el cálculo de engranajes rectos con un ángulo de presión de 20 grados que se ha denominado SolidG, tomando como referencia la norma AGMA para la determinación de los factores que influyen en los esfuerzos generados en los engranajes. Se analizaron varios elementos sometidos a diferentes condiciones de operación y se comparan con los resultados obtenidos mediante la aplicación del análisis de elementos finitos utilizado por el complemento de simulación de SolidWorks. Es una propuesta interesante como ejemplo de las capacidades que se pueden desarrollar en cuanto a la automatización de proceso de diseño, la reducción de los tiempos y el aumento en la productividad.

\section{RAUMPLAN Y EL VALOR COMPOSITIVO} DE LA GEOMETRÍA BASADA EN EL CUBO. Yannette Díaz Umaña, Mawency Vergel Ortega Julio Alfredo Delgado Rojas. Artículo

de investigación. Hace parte de los resultados de la investigación "Aproximación teóricometodológica al modelo de convergencia en Trabajo Social, aportes y desafíos al desarrollo de la profesión en el siglo XXI". En los resultados que se presentan, se destaca la categoría de renovación permanente en la formación disciplinar, desde donde se priorizaron las siguientes subcategorías: formar en la relación global y local, la importancia de articular saberes, el desafío de la investigación formativa y la necesidad del pensamiento crítico.

USO DE AULAS VIRTUALES EN CONTABILIDAD:UNESTUDIOCOMPARATIVO MODALIDAD DISTANCIA Y PRESENCIAL. Nataly Marcela Muñoz Murcia, David Andrés Camargo Mayorga, Jennifer Lorena Gómez Contreras, Universidad Militar Nueva Granada. Artículo de investigación. Analiza el uso de una de las tecnologías más utilizadas para mediar la educación, las aulas virtuales (ambientes virtuales de aprendizaje). Se concluye que se debe hacer un uso más efectivo de las aulas virtuales articulando nuevas formas didácticas y pedagógicas, lo cual requiere mayores procesos de formación y acompañamiento a docentes por parte de las instituciones.

\section{GEOMETRÍA, GRAVEDAD Y PATRIMONIO.} TRES VARIABLES DEL CAIXA FORUM MADRID . Yannette Díaz Umaña, Mawency Vergel Ortega Julio Alfredo Delgado Rojas. Artículo de investigación. Desarrolla una serie de estrategias que buscan alterar la percepción de los atributos en la relación entre la carga y el soporte. En la rehabilitación y ampliación de la antigua central eléctrica del Mediodía, un edificio patrimonial que data de 1899, la estructura en mampostería de ladrillo y hormigón debe conciliar la materia en equilibrio estático, evitando el colapso y preservando su vida útil. De ahí, el edificio en forma prismática rectangular se propone suspendido en el aire permitiendo el paso de los peatones por debajo él. De este modo se percibe la gravedad como una característica geométrica del espaciotiempo. Bajo estos conceptos, la espacialidad de Caixa Fórum es interesante por cuanto los muros portantes de la antigua fábrica pasan a ser una envoltura suspendida, que le dan una innegable corporeidad llamativa, firme y voluminosa. Esta caracterización le aporta una belleza particular, radicada precisamente en la experiencia que brindan las superficies coloridas y táctiles aproximando el paseo del eje Recoletos-Prado, en Madrid a un paseo cinestésico.

ABORDAJE Y CIERRE DELOS PROCESOS DE ENSEÑANZA Y APRENDIZAJE CON LOS NIÑOS Y LAS NIÑAS EN SITUACIÓN DE DISCAPACIDAD COGNITIVA EN EL AULA COMÚN DE SEGUNDO . Laura Nataly Galvis Velandia, Maritza Carolina Jaimes Márquez, Milton Alier Montero Ferreira. Artículo de investigación. Dirigido a generar fundamentos teórico-procedimentales para la atención de los aprendizajes actitudinales en torno a los 
procesos de enseñanza y aprendizaje de la niñez con discapacidad cognitiva del grado segundo en dicha institución, a partir de la caracterización en el abordaje y cierre de los procesos de enseñanza y aprendizaje con esta población en condiciones especiales.

Los hallazgos muestran en sus resultados que una vez aplicada la metodología en la triangulación de la información se determinaron los hallazgos en los cuales se identifica, que los estudiantes de segundo grado del colegio, según sus capacidades de aprendizaje, requieren una atención diferenciadora, pero integradora en los procesos pedagógicos de enseñanza.

\section{PROMOCIÓN Y ARTICULACIÓN DE REGIS-}

TROS SEMIÓTICOS DE REPRESENTACIÓN EN LA ENSEÑANZA DE LA FÍSICA. Raúl Prada Núñez, Audin Aloiso Gamboa Suarez, William Rodrigo Avendaño Castro. Artículo de investigación. Reporta los resultados derivados de un estudio adelantado con docentes que orientan Física en el nivel de Educación Secundaria y Media, con el fin de caracterizar los recursos didácticos que habitualmente implementan en su proceso de enseñanza. Los resultados permiten evidenciar el uso recurrente por parte de los docentes de situaciones mecánicas que dista de la solución real de problemas, por lo que siempre hacen uso de los siguientes registros semióticos de representación y en ese orden siempre: lenguaje natural, expresión algebraica y representación gráfica. Dado que el proceso es siempre en esa dirección, limita el entendimiento de conceptos y aprendizajes de los conceptos de Física necesarios tanto en el entorno académico con en su cotidianidad. 


\section{REFERENCIAS BIBLIOGRÁFICAS}

Ahumada, M A (2021). APUESTAS DESDE LA EDUCACIÓN POPULAR. Saberes y Sabidurías Pedagógicas: Encuentro de la palabra y las experiencias educativas. Revista Boletín Redipe, 10/12

Arboleda, JC (2021). PRESENTACIÓN.

TRANSMODERNIDAD, HERMENÉUTICA Y EDUCACIÓN. Revista Boletín Redipe, 10/10, pgs 2237

Duque, C A. EL GIRO CONSERVADOR EN LA OBRA DEL FILÓSOFO COLOMBIANO SANTIAGO CASTRO GÓMEZ, Revista Boletín Redipe, 10/10, pgs 50-64

Betancourt- Perez (2021). LA SUBJETIVIDAD $Y \quad L A$ INTERSUBJETIVIDAD: DOS VECTORES EN LA EVALUACIÓN COMO FENÓMENO ANTROPOLÓGICO. Revista Boletín Redipe, $10 / 12$

Cardona- Rivas Trujillo - Ramírez (2021). LOS MODELOS DIDÁCTICOS EN LA FORMACIÓN DE DOCENTES UNIVERSITARIOS, LA EMERGENCIA DE LOS SABERES-OTROS (TECNOLÓGICO Y AMBIENTAL) EN PANDEMIA DE LA COVID-19. Revista Boletín Redipe, 10/12

Ocaña, Díaz y Mejía (2021). DECOLONIZAR LAS CIENCIAS DE LA EDUCACIÓN: PEDAGOGÍA, ALTERSOFÍA Y HACER EDUCATIVO DECOLONIAL. Revista Boletín Redipe, 10/12 